\title{
NECK LEAF 1, a GATA type transcription factor, modulates organogenesis by regulating the expression of multiple regulatory genes during reproductive development in rice
}

\author{
Liping Wang ${ }^{1,3}$, Hengfu Yin ${ }^{1}$, Qian Qian ${ }^{2}$, Jun Yang ${ }^{1}$, Chaofeng Huang ${ }^{1}$, Xiaohe Hu${ }^{1}$, Da Luo ${ }^{1}$ \\ ${ }^{I}$ National Key Laboratory of Plant Molecular Genetics, Institute of Plant Physiology and Ecology, Shanghai Institutes for Bio- \\ logical Sciences, Graduate School of the Chinese Academy of Sciences, Chinese Academy of Sciences, Shanghai 200032, China; \\ ${ }^{2}$ China National Rice Research Institute, Chinese Academy of Agricultural Sciences, Hangzhou 310006, China
}

In the monocot rice species Oryza sativa L., one of the most striking morphological processes during reproductive development is the concurrence of panicle development with the sequential elongation of upper internodes (UPIs). To elucidate the underlying molecular mechanisms, we cloned the rice gene NECK LEAF 1 (NL1), which when mutated results in delays in flowering time, smaller panicles with overgrown bracts and abnormal UPI elongation patterns. The $N L 1$ gene encodes a GATA-type transcription factor with a single zinc finger domain, and its transcripts are detected predominantly in the bract primordia, which normally degenerate in the wild-type plants. Overexpression of $N L 1$ in transgenic plants often gives rise to severe growth retardation, less vegetative phytomers and smaller leaves, suggesting that NL1 plays an important role in organ differentiation. A novel mutant allele of PLASTOCHRON1 (PLA1), a gene known to play a key role in regulating leaf initiation, was identified in this study. Genetic analysis demonstrated an interaction between $n l 1$ and pla1, with $N L 1$ acting upstream of PLA1. The expression level and spatial pattern of PLA1 were found to be altered in the nl1 mutant. Furthermore, the expression of two regulators of flowering, $H d 3 a$ and $O s M A D S 1$, was also affected in the $n l 1$ mutant. On the basis of these findings, we propose that NL1 is an intrinsic factor that modulates and coordinates organogenesis through regulating the expression of PLA1 and other regulatory genes during reproductive development in rice.

Keywords: elongation of upper internodes, organogenesis, panicle development, phase transition, NECK LEAF 1, GATAlike transcription factor

Cell Research (2009) 19:598-611. doi: 10.1038/cr.2009.36; published online 31 March 2009

\section{Introduction}

The aerial body of flowering plants usually develops in a continuous and repetitive manner during postembryonic development, and various stages or phases can be identified by characteristic morphological traits $[1,2]$. During phase transitions, organogenesis normally occurs and with prominent morphological alterations, giving

Correspondence: Da Luo

Tel: +86-21-54924108; Fax: +86-21-54924015

E-mail: dluo@sibs.ac.cn

${ }^{3}$ Present address: Plant Biotechnology Institute, National Research Council of Canada, 110 Gymnasium Place, Saskatoon, SK S7N 0W9, Canada Received 21 April 2008; revised 16 June 2008; accepted 16 October 2008; published online 31 March 2009 rise to organ size, shape and identity. Both conserved and divergent mechanisms among species are expected to play key roles in the control of meristem identity and organogenesis, and account for the molecular basis of immensely divergent natural plant architectures $[1,3]$. The rice species Oryza sativa L., a model monocot plant with its entire genome sequence being available, presents a unique opportunity to dissect key components involved in the control of distinct morphological processes, which could affect some important agronomic traits.

Various developmental stages in rice have previously been investigated, and a number of key regulators have been identified [4-6]. Morphological development in the vegetative phase is characterized by repetitive vegetative phytomers consisting of leaves, nodes, unelongated internodes and axillary buds. Transition from the juvenile to 
the adult phase can be distinguished by leaf morphology, stem structure and size of shoot apical meristem (SAM) $[4,5,7]$. The moril mutant reiterates early vegetative growth (second leaf stage) and fails to enter the adult phase, suggesting that MORI1 plays an important role during this transition [7]. The PLASTOCHRON1 (PLA1) gene encodes a cytochrome P450 monooxygenase and acts as a timekeeper of leaf initiation. Mutations in PLA1 result in shortened leaf plastochrons and a prolonged vegetative program [8-10]. PLA2, another key regulator, encodes an MEI2-like RNA-binding protein that independently controls the rate of leaf initiation; the pla2 mutant displays a plal-like phenotype [11]. In response to both environmental conditions and internal factors, SAM identity transitions between the vegetative and reproductive phases in rice, acquiring the inflorescence identity to give rise to the panicle. The transition between the vegetative and reproductive phases in rice is thought to require expression of both PLA1 and PLA2 for proper termination of vegetative development $[10,11]$.

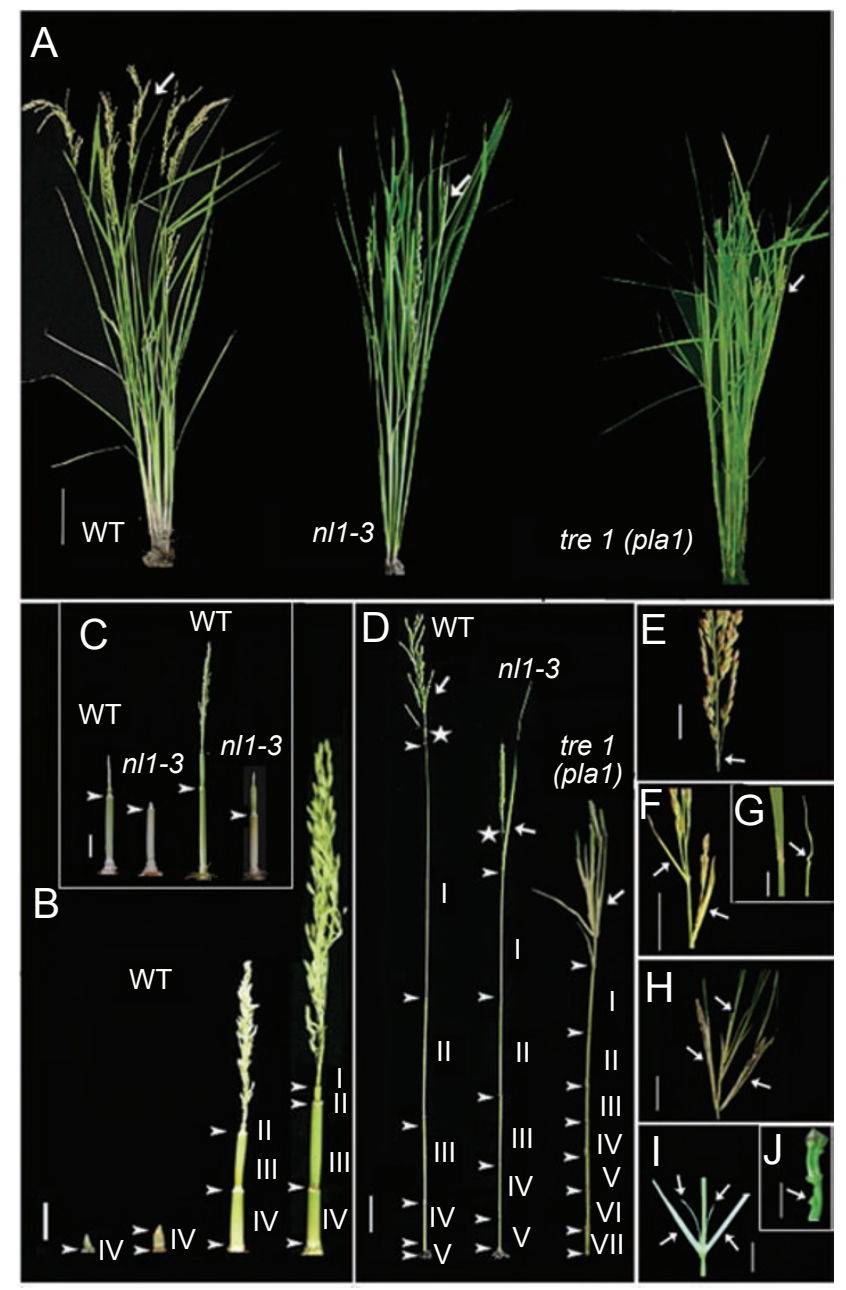

During the reproductive phase, repeated developmental modules in the panicle begin to differ morphologically from those in the vegetative phase. Floral differentiation gives rise to the rachis branches and spikelets, and there is no visible leaf or bract owing to immediate degeneration of the bract primordia. Upon a shift from vegetative to reproductive growth, four or five upper internodes (UPIs) in some vegetative phytomers sequentially elongate from the basal end (UPI-IV or UPI-V, depending on the genotype of the cultivated rice) to the uppermost UPI (designated UPI-I), concomitant with panicle development (Figure 1B) [4, 5]. Furthermore, the longest UPI is always designated as UPI-I and the shorter UPIs extend basally (Figure 1D). In fact, the heading in rice (i.e. the UPI-I rapidly elongates while the panicle concomitantly emerges from the flag leaf, the last leaf or the uppermost leaf in a culm), is a characteristic phenomenon which has attracted attention in both basic research and rice breeding.

Most studies have thus far been focused on quantita-

Figure 1 Mutants affecting the pattern formation during panicle development. (A) Wild-type and mutant plants after the heading: wild type (Zhonghua 11, left), n/1-3 mutant (middle) and tre1 (pla1) mutant (right). Arrows indicate the panicles in wild type, sheathed panicle in $n / 1-3$ mutant, clustered shoots and small panicles in tre1 (pla1); bar $=5 \mathrm{~cm}$. (B) Synchronicity of panicle developmental stages with UPI initiation/elongation in wild type; bar $=1 \mathrm{~cm}$. The arrowheads indicate the nodes, which separate the sequential elongated UPIs; UPI-I, UPI-II, UPI-III and UPI-IV stand for different UPIs from the uppermost to the basal vegetative phytomers. (C) Comparison of UPI initiation/elongation in wild type and $n / 1-3$ at two developmental stages to show the different synchronism between the developments of panicle and UPIs; bar $=2 \mathrm{~cm}$. (D) A culm of wild type (left), n/1-3 mutant (middle) and tre1/pla1 (right), vegetative leaves being removed. The asterisks indicate the positions of basal primary branch, bar $=5 \mathrm{~cm}$. (E-F) Panicles of wild type and $n / 1-3$; bars $=2 \mathrm{~cm}$. (E) wild-type panicle with no visible bracts (arrow); $(\mathrm{F})$ n/1-3 panicle with overgrown bracts (arrows) on the base of the primary rachis branches. (G) Comparison of normal vegetative leaf in wildtype (left) and overgrown bract of $n / 1-3$ (right); bar = $1 \mathrm{~cm}$. Arrow indicates the boundary between leaf blade and sheath. (H-J) Structure of panicle, node and stem in tre. (H) A primary rachis branch of tre: clustered shoots and overgrown bracts (arrows) are shown. (I) An abnormal node of tre with a pair of leaves (lower arrows) and a pair of axillary shoots (upper arrows) in contraposition. (J) A piece of disorganized stem of tre with the internodes and nodes (arrow) in abnormal position. Bar $=5 \mathrm{~cm}$ in $\mathrm{H}$; bar $=$ $2 \mathrm{~cm}$ in $\mathrm{I}$; bar $=1 \mathrm{~cm}$ in $\mathrm{J}$. 
tive trait loci in the control of heading time in rice, and several key components, such as $H d 1, H d 3 a$ and Ehd1, have been cloned [12-14]. $H d 1$ and $H d 3 a$ are orthologs of CONSTANS (CO) and FLOWERING LOCUS T (FT) in Arabidopsis; OsGI and OsMADS50 are the Arabidopsis counterparts of $G I$ and SOC1 [12, 13, 15], indicating that key components in the control of floral development are well conserved among both dicots and monocots. In addition, overexpression of OSMADS1 in transgenic tobacco plants led to dramatically early flowering, as well as reduced apical dominance, suggesting the involvement of OSMADS1 in the regulation of flowering time [16].

Heading in rice occurs in synchrony with panicle organogenesis and UPI elongation, suggesting that signaling occurs between the panicle and UPIs during the reproductive phase [17]. Identification of regulators of UPI elongation and floral development should provide clues to underlying genetic factors that coordinate organogenesis in both vegetative phytomers and panicles. Various loci affecting UPI elongation in rice have been identified as dwarf mutants, which can be categorized into several groups based on the distinct defects in UPI elongation [18]. Some mutants display shortening of one or two specific UPIs, while others proportionally affect the length of all UPIs. One mutant called $s d l$ or an allele of OsGA20ox2 encoding a key enzyme for the gibberellic acid (GA) synthesis has been widely utilized in rice breeding for its semidwarf phenotype and is well known as a key factor for the green-revolution crop [19]. Both genetic and molecular studies of these two mutant classes have helped to identify key regulatory genes that encode components in phytohormone pathways, such as GA and brassinosteroid (BR) pathways [16, 20-23]. Consistently, twenty-nine genes are identified from rice genome, which encode the putative GA metabolic enzymes, and some mutants of these genes showed dwarf or semidwarf phenotypes with reduced length of all UPIs [24]. On the other hand, a homeodomain protein OSH15 was also found to be important in regulating epidermal cells and arranging small vascular bundles (VBs) of internodes [25]. These data support the notion that a sophisticated genetic network involving multiple signaling pathways modulates UPI elongation patterns. Another group of dwarf mutants, including $n l 1$, affect differential UPI elongation and possess UPIs with less differential lengths. Furthermore, nll displays a conspicuous floral phenotype of smaller panicles with overgrown bracts, from which the mutation neck leaf received its name [26] (Figure 1D and 1F). In nll mutants, an abnormal heading is observed, as UPI-I is shorter and the spathe-like neck leaf encases the panicle. Thus, NECK LEAF 1 (NL1) likely modulates and coordinates organogenesis in both vegetative phytomers and panicles during reproductive development in rice.

In this study, we cloned the $N L 1$ gene and identified a new allele of pla1. NL1 encodes a GATA-type transcription factor expressed predominantly in the bract primordia. Ectopic expression of NL1 produces less vegetative phytomers, smaller leaves and panicles, suggesting that NL1 plays an important role in regulating organ differentiation. Genetic analysis revealed that plal is epistatic to $n l 1$, and the PLAl expression pattern is altered in the $n l l$ mutant. Expression of $N L 1$, however, is maintained in pla1 mutants, suggesting that $N L 1$ may act upstream of PLA1 during panicle development. Furthermore, expression of two flowering regulators, $H d 3 a$ and $O s M A D S 1$, is downregulated in the $n l l$ mutant. Based on genetic analyses and gene expression patterns, we propose that the main function of NL1 is to modulate various pathways to coordinate organogenesis in both vegetative phytomers and panicles during reproductive development in rice.

\section{Results}

Mutants giving rise to defects in pattern formation during panicle development

In the wild-type Zhong Hua 11 (ZH11) japonica cultivar, 13 phytomers usually develop at the vegetativereproductive transition. As the development of panicle meristem starts, four UPIs also begin to sequentially elongate (Figure 1B). To identify key components that regulate organogenesis during transition from the vegetative to the reproductive phase, two types of mutants were analyzed (Figure 1A). The mutants displayed excessive vegetative growth with altered phytomer patterns and defects in panicle development to differing extents (Figure $1 \mathrm{~A}$ and $1 \mathrm{D})$.

Genetic analysis was conducted to characterize the mutants, and the relevant loci were mapped using molecular markers. Four mutants (named tree rice) with strong pleiotropic phenotypes displayed equal lengths of elongated internodes on multiple vegetative phytomers. All mutants were single recessive with genetic loci closely linked to the PLA1 gene, a previously identified developmental regulator [8]. One putative plal mutant, tre1, was analyzed in detail. tre 1 carries a one-nucleotide deletion in the second exon, giving rise to a truncated nonfunctional protein and the abnormal mutant phenotype (Supplementary information, Figure S1). trel and other putative plal mutants display late flowering, excessive vegetative growth of 21 vegetative phytomers, 7 elongated UPIs and overgrown bracts with a cluster of shoots in the panicle (Figure 1A, 1D and 1H). Several putative pla1 mutants were observed to display a symmetric ab- 
normity in some vegetative phytomers: a pair of leaves and a pair of axillary buds were in contraposition to the node, and stems were disorganized, with abnormal node and internode positions (Figure 1I and 1J). These data suggest that the PLA1 gene may act directly in the control of phytomer development and determine the basic structure of developmental modules.

Because other mutants were found to be allelic to a classic morphological locus, NL1 (Figure 1A and 1D) [16], we designated these mutants as $n l 1-1, n l 1-2$ and $n l 1-3$. The nll mutants possess one extra vegetative phytomer with five UPIs (Figure 1D) and exhibit a delayed transition (by several days) from vegetative to reproductive growth when compared with wild-type plants (Supplementary information, Figure S2). Overgrown bracts at the base of the panicle most distinctly characterize the $n l l$ phenotype and explain how neck leaf was originally named. One or two large bracts generally develop at the basal node of the panicle, and several smaller bracts are visible at the base of primary branches (Figure 1F), in contrast to wild-type plants that bear no visible bracts (Figure 1E).

\section{Developmental UPI patterns and panicles are altered in the nll mutant}

SAM development in both the wild type and the nll-3 mutant was analyzed using scanning electron microscope (SEM). No detectable differences were observed between the wild type and the mutant until initiation of the first bract primordium in the panicle meristem. In the wild-type plant, the first bract primordium reaches its maximum size and begins to degenerate at the late stage of primary branch development (Figure 2A). However, at a similar developmental stage in the nll-3 mutant, the first and second bracts continue to grow and begin wrapping around the panicle (Figure 2B), a characteristic of vegetative leaf primordia. Conspicuous neck leaves at the basal end of the panicles in the different $n l l$ mutants contain both leaf blades and sheaths, but are much smaller than vegetative leaves (Figure $1 \mathrm{G}$ ). However, the ligule and auricle normally found at the boundary between the blade and sheath in vegetative leaves are not present in the neck leaves (Figure $1 \mathrm{G}$ ). In the $n l 1$ mutant, the panicle is smaller (Figure 1D) and bears fewer spikelets (70.8 \pm 7.79 in the $n l 1$ mutants versus $94 \pm 10.82$ in wild-type plants) with a reduced number of primary branches $(6.8$ \pm 0.75 versus $8.3 \pm 0.58$ ) (Supplementary information, Figure S3). These data indicate that the developmental pattern of the panicle is altered in $n l l$ mutants.

The $n l 1$ mutant phenotype was described as a type of dwarf mutant with the spathe-like neck leaf at the base of the panicle [16]. Indeed, despite excessive vegetative development and an extra phytomer in $n l l$ mutants, plant height is reduced compared with that of wild type (Figure $1 \mathrm{~A}$ and 1D). Additionally, the final lengths of the elongated internodes for $n l 1-3, n l l-2$ and their progenitors (ZH11 and ZF802) were analyzed. The $n l 1$ mutants were found to have reduced UPI-I lengths and an elongated basal internode compared with the wild type (Figures 1D and $2 \mathrm{H}$; Table 1). Thus, $n l 1$ mutants exhibit an isometric elongation pattern, in contrast with the wild-type case in which UPI-I normally represents more than $50 \%$ of the total culm length and the lengths of lower internodes gradually decrease (Table 1). Both the neck leaf and abnormal UPI elongation patterns give rise to the panicle extrusion defect in $n l l$ mutants (Figure 1A and 1D). In the wild-type plant, elongation commences with the most
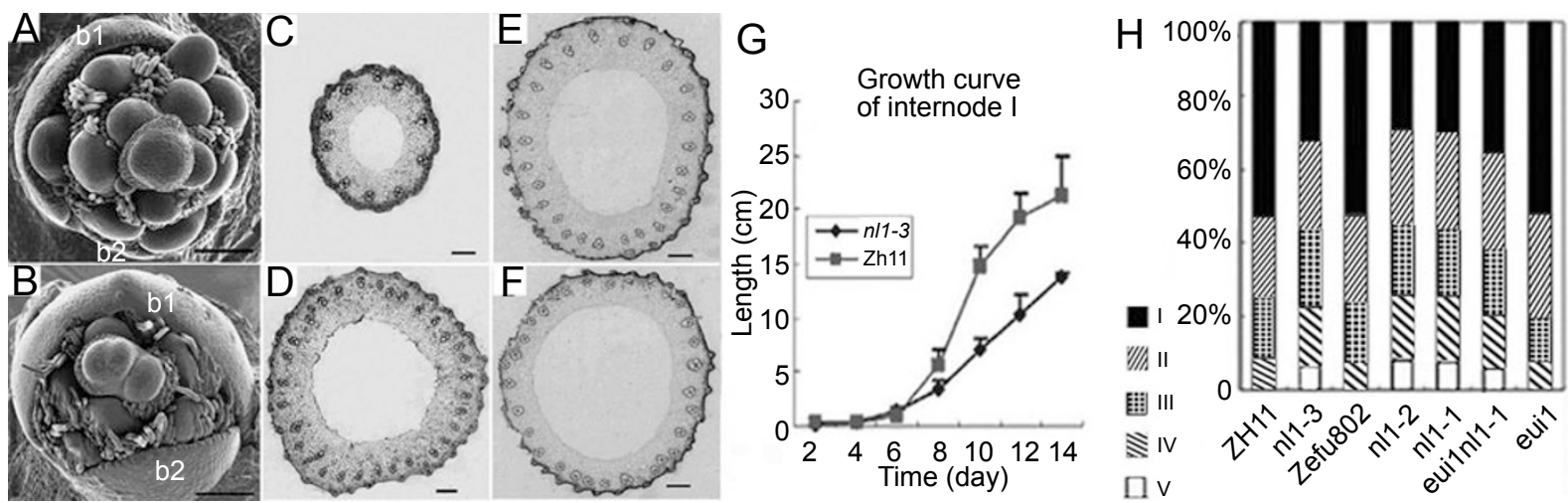

Figure 2 Bract development and pattern of UPIs in wild type and mutants. (A-B) Panicles of wild type ZH11 (A) and $n / 1-3$ (B) at the stage when primary rachis-branch primordia differentiate. Bars $=50 \mu \mathrm{m}$. b1, the first bract primordium; b2, the second bract primordium. (C-F) Cross-sections of internodes of wild type (C, E) and n/1-3 mutant (D, F). (C-D) The uppermost internode I; (E-F) the second internode; bars = $200 \mu \mathrm{m}$. (G) The growth curve of internode I between wild type and $n / 1-3$. (H) Length proportion of UPIs in different genetic backgrounds. 
Table 1 Comparisons of length of each elongated internode between wild type and mutant

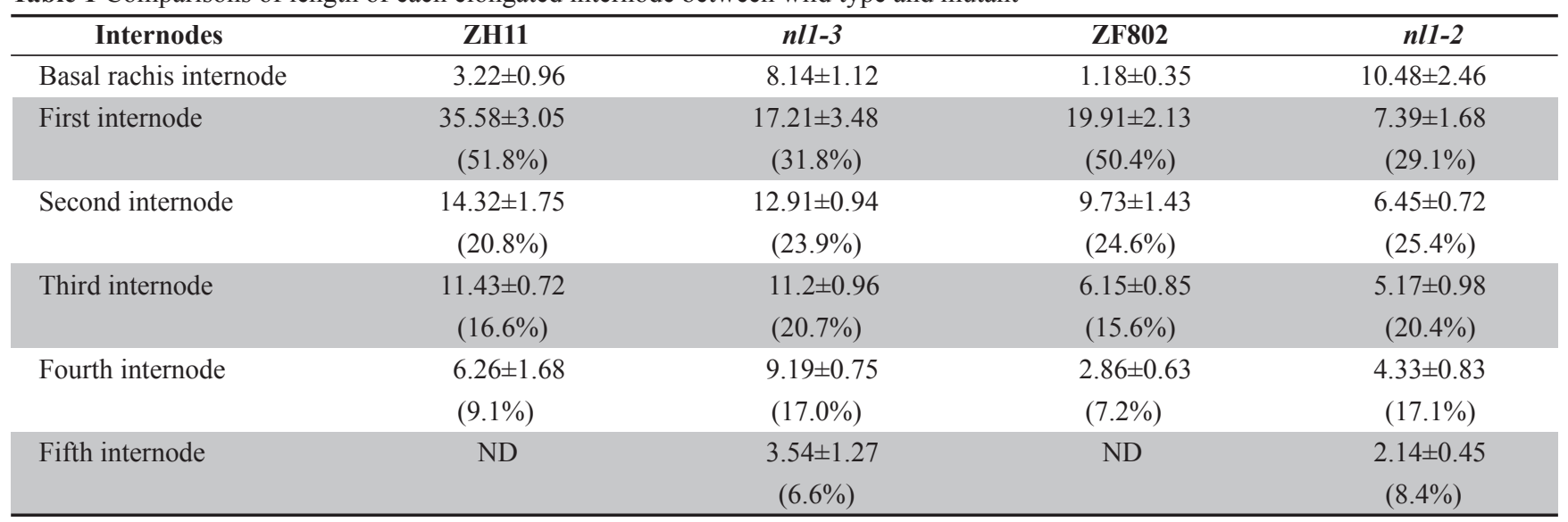

Length of the upper four or five elongated internodes and the basal rachis internode were measured. The length is shown in centimeters. Data are averages of 15 main culms ( \pm S.D.). The relative contribution of each elongated internode to the total culm is shown in the brackets. ND, not detected.

Table 2 The comparisons of vascular number between wild type (ZH11) and mutant (nll-3)

\begin{tabular}{cccccccccccc}
\hline & I-I & I-s & II-I & II-s & III-I & III-s & IV-I & IV-s & V-I & V-s \\
\hline ZH11 & $8.5 \pm 1.3$ & $21.8 \pm 1.3$ & $30.0 \pm 2.7$ & $27.5 \pm 2.4$ & $29.0 \pm 2.7$ & $28.8 \pm 3.4$ & $27.5 \pm 1.7$ & $28.0 \pm 1.6$ & ND & ND \\
$n l 1-3$ & $27.0 \pm 2.4$ & $28.0 \pm 4.8$ & $30.0 \pm 2.2$ & $28.2 \pm 2.9$ & $30.8 \pm 0.9$ & $28.8 \pm 1.0$ & $29.8 \pm 1.0$ & $29.5 \pm 1.3$ & $30.5 \pm 1.7$ & $28.0 \pm 1.4$ \\
\hline
\end{tabular}

Data represent the numbers of large and small vascular bundles, which are denoted by 1 and s, respectively. ND, not detected. Data are averages of four main culms ( \pm S.D.). I, first internode; II, second internode; III, third internode; IV, fourth internode; V, fifth internode.

basal UPI and extends upward in synchrony with PB initiation and development of floral organs in the panicle, respectively (Figure 1B). However, this synchrony is altered in $n l l$ mutants, and panicle development occurs much later than in the wild type (Figure 1C). When UPII elongation curves are compared, the nll mutant curve differs from the typical S-type growth curve in the wild type (Figure 2G). Therefore, UPI elongation patterns in nll mutants are impaired with the development of the panicle in comparison with that in the wild type.

In wild-type rice, the stem widths and vascular patterns differ between the upper and basal elongated internodes (Figure 2C and 2E). Histological sections of internodes demonstrate that two rings of VBs are present within UPIs. VBs located in the inner ring are larger than those in the outer ring. In wild-type plants, there are far fewer VBs within the inner ring of UPI-I than in the outer ring, whereas the number of large and small VBs is nearly equivalent in the most basal UPIs (Figure 2C and 2E; Table 2). In the $n l 1$ mutant, however, stem widths of the upper and basal internodes are similar, and the number of large and small VBs in UPI-I increase to become nearly equivalent like that of basal UPIs (Figure 2D and 2F; Table 2). Thus, developmental UPI and panicle patterns were altered in $n l l$ mutants, and the growth rate and synchrony of both vegetative and reproductive organogenesis are affected, suggesting that NL1 plays an important role in monitoring and coordinating organogenesis during panicle development.

Malfunction in the nl1 mutant is independent of the photoperiodic and GA signaling pathways

Stability of the $n l l$ phenotype has been examined in detail. When the nll-3 mutant was grown in long-day and short-day conditions, 14 and 12 vegetative phytomers, respectively, developed. The wild-type plant, however, produced 13 and 11 vegetative phytomers, respectively. Therefore, the additional vegetative phytomer in $n l l$ mutants developed independently of either long-day or short-day conditions, as did the other properties conferred by the $n l l$ mutant. Furthermore, when an indica introgression line with $n l 1-2$ allele was analyzed, the $n l l$ phenotype was found to be stable in both indica and japonica cultivar genetic backgrounds, which possess different photoperiodic responses (data not shown).

Rice dwarf mutants with shorter UPI lengths often result from a deficiency in GA signaling. Because $n l l$ mutants also display dwarf-like phenotypes with shorter UPIs, we analyzed whether the function of $N L 1$ is linked with the GA signaling pathway. Treatment with $\mathrm{GA}_{3}$ 
caused the length of all UPIs to increase in the $n l l$ mutant without altering the abnormal length patterns observed among different UPIs (Supplementary information, Figure S4). To test the effects of endogenous GA phytohormone in the $n l l$ mutant, we constructed a double mutant by crossing $n l 1-2$ with euil, a mutant that accumulates a mass of biologically active gibberellins in the UPIs [23, 27]. The $n l 1-2$ euil double mutant displayed an additive effect: All UPI lengths increased, but the isometric pattern was maintained (Figure $2 \mathrm{H}$ ), suggesting that $N L 1$ acts independently of the GA signaling pathway.

\section{NL1 encodes a GATA-type zinc finger protein}

To clone the NL1 gene, two mapping populations were constructed and a map-based cloning strategy was used. The NL1 locus was mapped to the distal region of the long arm of rice chromosome 5 by mapping 188 plants with the $n l l$ mutant phenotype and was subsequently refined to a smaller $7 \mathrm{~kb}$ region using a total of $1253 \mathrm{~F}_{2}$ mutant plants (Figure 3A).

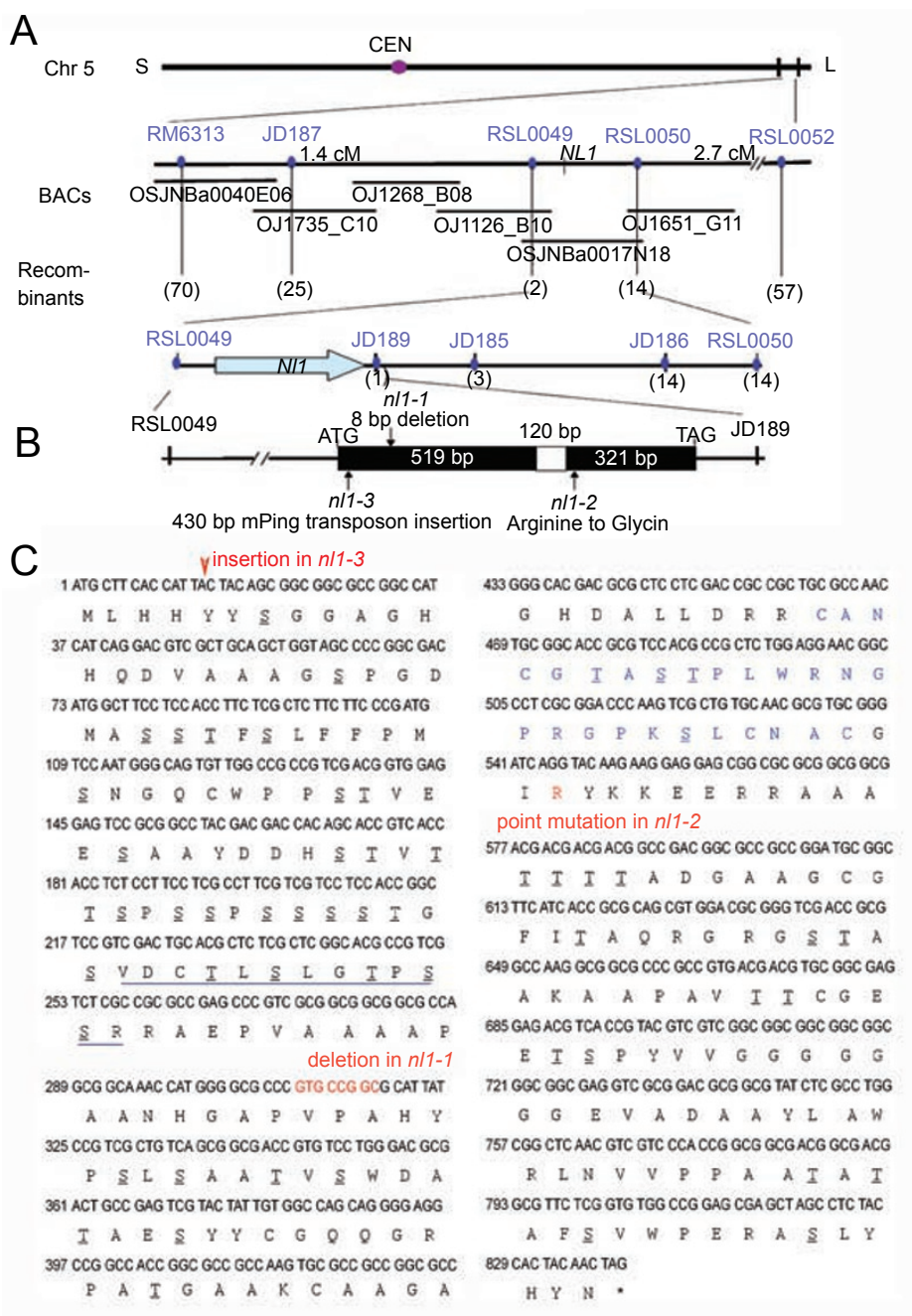

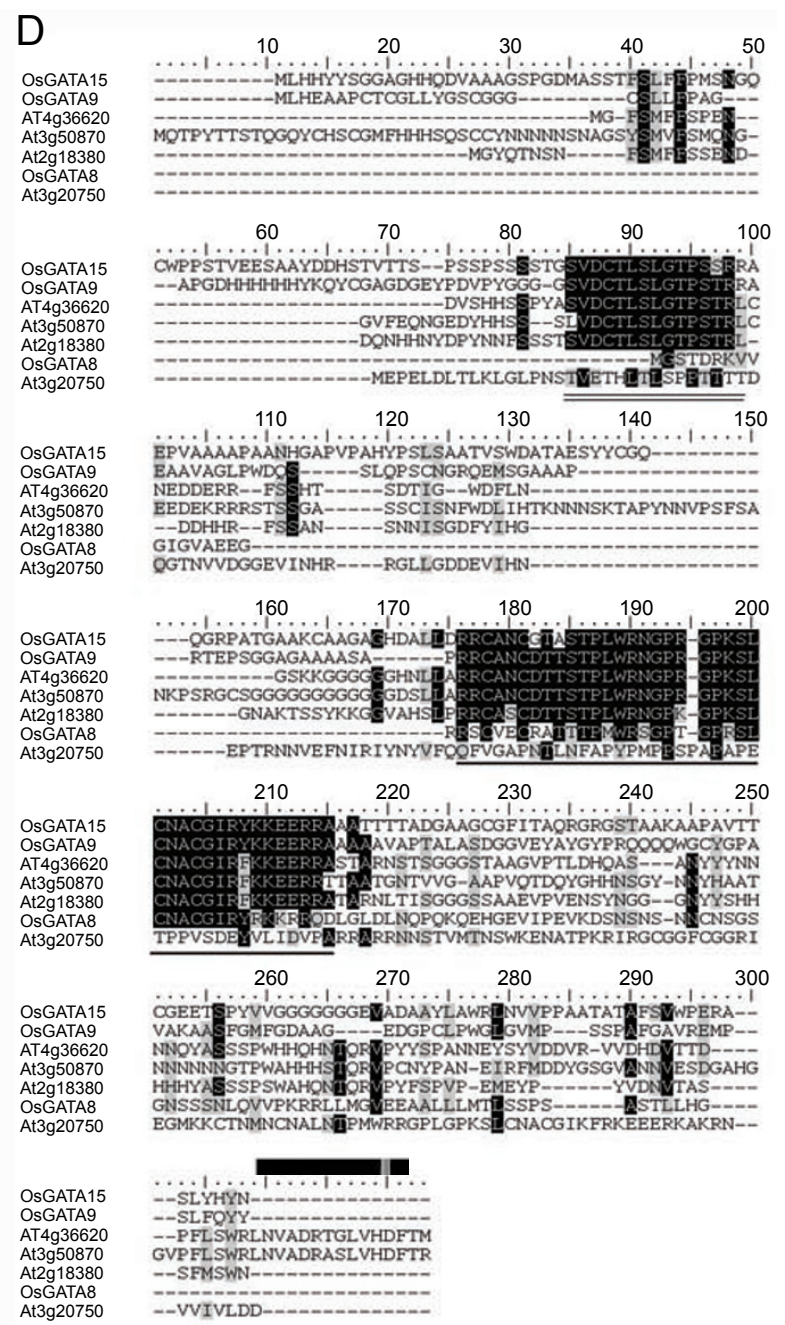

Figure 3 Molecular cloning of NL1 gene and analysis of NL1 protein sequence. (A) The NL1 gene is mapped to a 7-kb genomic region between two SSR markers RSL0049 and JD189. The centromere is indicated (CEN). The code/number of consecutive BAC clones are shown in black and the molecular markers are shown in blue. The number of recombinants is shown in parenthesis. (B) NL1 gene structure. NL1 consists of two exons (filled rectangles) and one intron (open rectangle). Mutations being identified in $n / 1-1, n / 1-2$ and $n / 1-3$ are indicated by arrows. (C) NL1 cDNA and putative amino acid sequences. The C-X2-C-X18-C-X2-C zinc-finger domain and N-terminal conserved domain are shown in bold blue letters and blue lines. Red letters indicate mutations that occurred in three alleles. Serine and threonine residues are underlined. (D) Alignment of GATA-like zinc finger proteins. Seven GATA-like zinc finger proteins of class B (three from rice and four from Arabidopsis) are aligned. Identical residues are shaded in black. The C-X2-C-X18-C-X2-C zinc-finger domain and N-terminal conserved motif are underlined. 
Using the rice genomic database and the GENSCAN program, only one gene was annotated within this region, encoding a putative GATA-type zinc finger protein. The coding region and gene structure of the candidate $N L 1$ gene were confirmed by reverse transcription (RT)-PCR. Analysis of DNA sequences in the wild type and three nll mutants showed that the $n l 1-1$ mutant carries an 8-bp deletion in the first exon of the candidate gene, likely leading to a frameshift mutation and shorter peptide (Figure $3 \mathrm{~B}$ and $3 \mathrm{C}$ ). In the $n l 1-2$ mutant, an arginine-to-glycine (R182G) amino acid substitution was found within a conserved region followed by the zinc finger domain. In the $n l 1-3$ mutant, a 430-bp mPing MITE transposon [28] was inserted in the first exon, altering the coding region of the NL1 gene. Complementation tests were conducted, and a 6.2-kb genomic fragment containing the putative $N L 1$ gene (including the coding region, $5^{\prime}$ upstream sequence and $3^{\prime}$ downstream region) was transformed into an nl1-1 background. As a control, the pCAMBIA1301 vector was also transformed into nll-1. We found that the nl1-1 mutant phenotype was rescued in transgenic plants carrying the candidate gene (Figure 4A) and conclude that the candidate gene is NL1.

Sequence analysis showed that the NL1 gene consists of two exons and one intron (Figure 3B and 3C). It encodes a 279-amino acid protein (GenBank accession number DQ784546) with a C-terminal zinc finger domain and a highly conserved N-terminal motif (Figure 3D). The amino acid composition of the NL1 protein is similar to that of GATA-1 through GATA-4 in Arabidopsis, containing approximately $19.3 \%$ serine and threonine residues that are possibly phosphorylated (Figure 3C) [29]. NL1 has a single zinc finger motif (Cys-X-X-Cys(X)18-Cys-X-X-Cys), followed by 10 amino acids at its carboxyl-terminal tail (Figure 3C and 3D). Phylogenetic analysis demonstrates that there are 28 GATA factors in
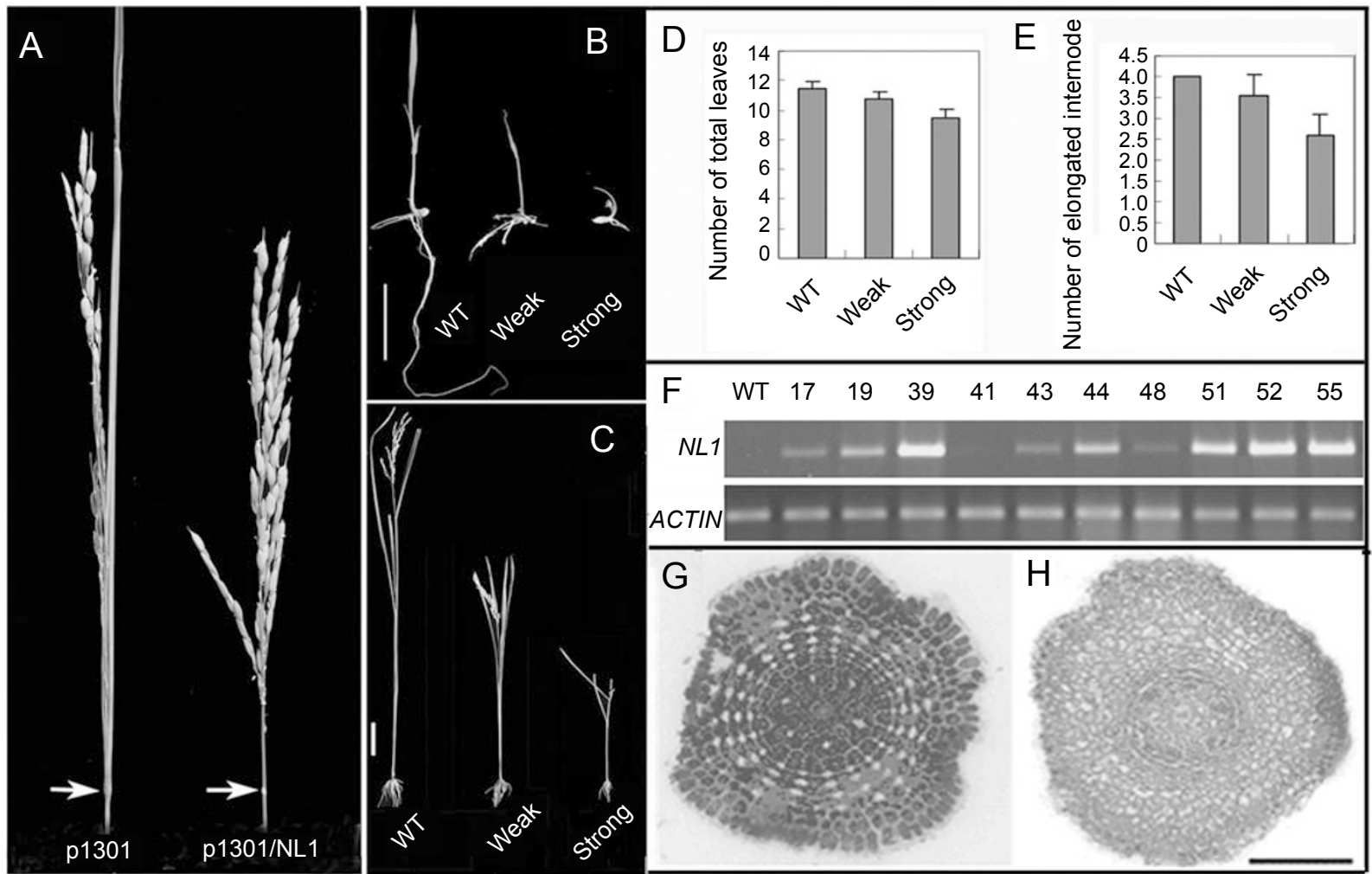

Figure 4 Complementation and ectopic expression of $N L 1$ in transgenic rice. (A) Panicles of $n / 1-1$ transgenic mutant carrying pCAMBIA1301 vector (left) and the mutant carrying complementary transgene, pCAMBIA1301/NL1 (right). The panicle node in transgenic plants is indicated (arrow), and the overgrown bracts are seen to disappear in the complementary transgenic plant (right). (B-C) Wild-type and retarded $T_{2}$ transgenic plants at the seedling stage $(B$, bar $=2 \mathrm{~cm})$, and at the heading stage $(C$, bar $=5 \mathrm{~cm})$. Transgenic plants with weak and strong phenotypes are shown. (D-E) The numbers of total leaves (D) and UPIs (E) in wild-type and transgenic plants with weak and strong phenotypes, $n=10$. (F) Ectopic expression levels of NL1 and transgene in wild-type and transgenic plants, the numbers stand for different individual transgenic plants in $\mathrm{T}_{0}$. (GH) Transverse section of root elongation zone from 10 day seedlings of wild type $(\mathbf{G})$ and the $35 S-N L 1$ transgenic plants $(\mathbf{H})$, bars $=200 \mu \mathrm{m}$. 
the rice genome and 29 in Arabidopsis; NL1 is identical to the previously described OsGATA 15 class B GATA factor [30]. According to gene structure and sequence homology, OsGATA15, OsGATA8, OsGATA9, as well as four Arabidopsis GATA factors constitute one cluster among the class B GATA factors [30]. Zinc-finger domains are conserved among these proteins by homology analysis, and five possess a conserved $\mathrm{N}$-terminal motif (Figure 3D).

NL1 is predominantly expressed in the bract primordia during panicle development

We investigated NL1 temporal and spatial expression patterns. NL1 was strongly expressed in young inflorescences and weakly expressed in the roots and elongating internodes as shown by RT-PCR analysis. No expression was detected in the vegetative SAMs, leaf blades and sheaths (Figure 5A). Furthermore, a marked reduction in expression was detected in the $n l 1-1$ mutant (Figure 5A), suggesting that functional NL1 protein is required for maintaining transcript levels. The NL1 mRNA localization in the wild-type plant was further examined by in situ hybridization. Upon transition from vegetative to reproductive growth, $N L 1$ transcripts were first detected in the bract primordium and then on the adaxial side of the mature bract (Figure 5B). During panicle development, NL1 transcripts were generally detected in all degenerated bracts and in the incipient bract primordia (Figure $5 \mathrm{C})$. Strong expression of $N L 1$ continued on the adaxial side of the first and second degenerate bracts until spikelet development commenced (Figure 5D). Therefore, $N L 1$ is predominantly expressed in the bract primordia
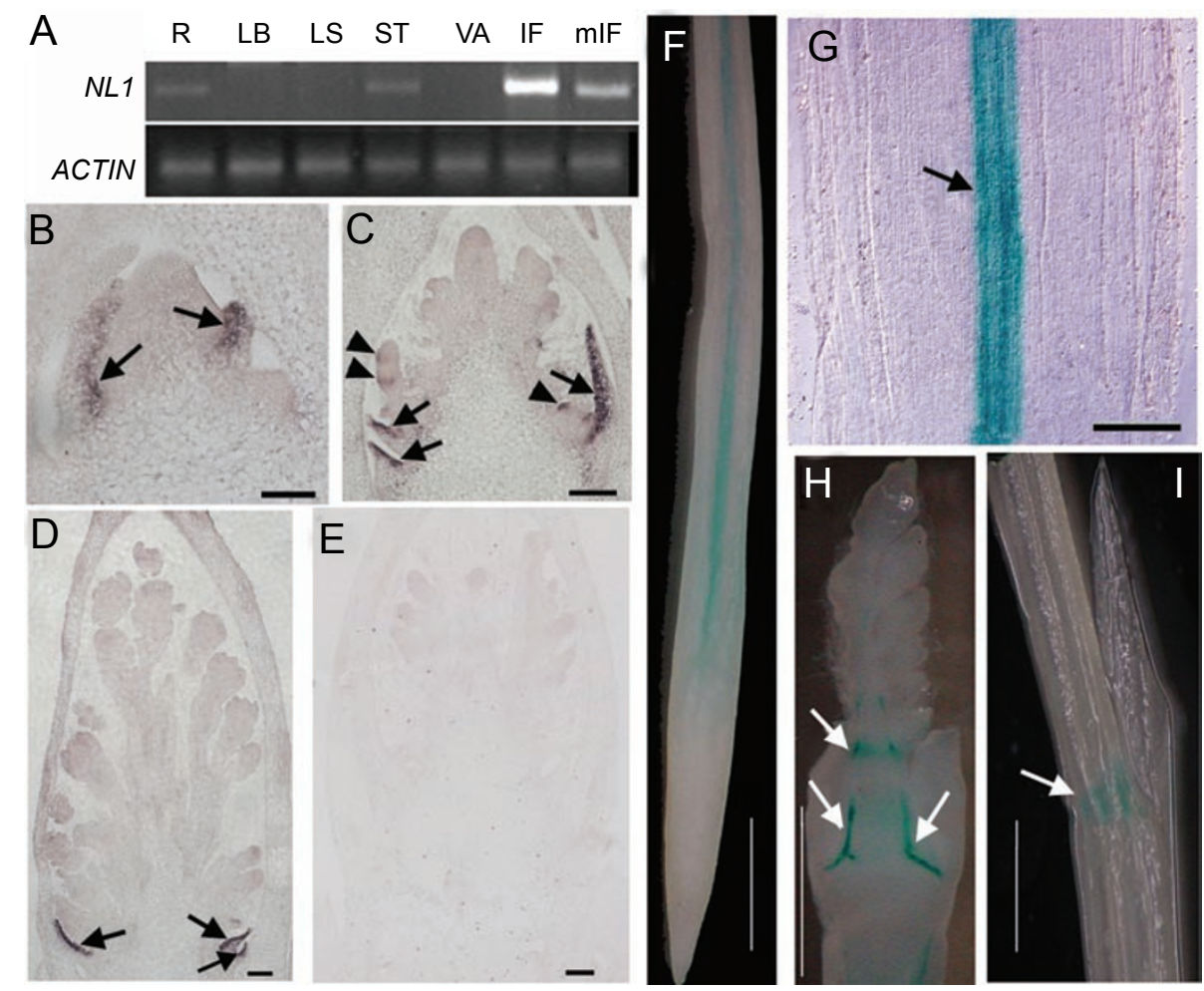

Figure 5 NL1 expression pattern. (A) RT-PCR analysis of NL1 expression. cDNA templates were prepared from root (R), leaf blade (LB), leaf sheath (LS), stem (ST), vegetative SAM (VA), inflorescence (IF) of wild type and inflorescence of $n / 1$-1 mutant (mIF). ACTIN was used as the endogenous reference control. PCR cycles for amplifying NL1 and ACTIN were 30 and 19 , respectively. (B-E) RNA in situ localization of $N L 1$; bars $=100 \mu \mathrm{m}$. (B) Longitudinal section of shoot apex at the transition from vegetative to reproductive phase. NL1 transcripts are detected the adaxial side of the first bract primordium (arrows, the dark staining). (C) Longitudinal section of a young panicle at stage when secondary branch primordia differentiate. NL1 transcripts are detected at the incipient bract primordia of secondary rachis branch (arrowheads), as well as at the adaxial side of the first and second bract primordia (arrows). (D) Longitudinal section of a panicle at stage when stamen and carpel primordia differentiate. NL1 transcripts can only be detected at the adaxial side of the first and second bract primordia (arrows). (E) Sense probe as a negative control. (F-I) GUS staining in the transformants with NL1 promoter-GUS. GUS staining is found in root stele (F-G), internode (H, shown by arrows) and the boundary between leaf blade and sheath $(\mathbf{I})$. Bar $=250$ $\mu \mathrm{m}$ in $\mathrm{G}$; bars $=0.5 \mathrm{~cm}$ in $\mathrm{F}, \mathrm{H}$ and $\mathrm{I}$. 
during panicle development. When the $N L 1$ promoter region (2 172-bp fragment extending from -2174 to -3 ) was fused to a $\beta$-glucuronidase (GUS) reporter gene, GUS expression was also detected in the VBs of elongating UPIs in the stele of roots within the elongation zones and in the junctions of leaf blades and sheaths (Figure $5 \mathrm{~F}-5 \mathrm{I}$ ), indicating that $N L 1$ is preferentially expressed in regions of secondary growth or elongation. However, GUS staining was not clearly found at the degenerate bracts (Figure $5 \mathrm{H}$ ), suggesting either that the NL1 promoter-GUS construct may not accurately mimic endogenous $N L 1$ gene expression or that the GUS protein was not actively synthesized in the degenerate bracts.

Ectopic NL1 expression results in growth retardation in transgenic rice

To further investigate $N L 1$ function, transgenic rice plants with constitutive NL1 expression under the cauliflower mosaic virus $35 \mathrm{~S}$ promoter were created and analyzed. Among 37 positive transgenic lines, 34 displayed abnormal phenotypes. RT-PCR analysis confirmed ectopic $N L 1$ expression levels in the transgenic plants. A strong correlation was observed between the abnormal phenotype and $N L 1$ levels. Transgenic plants with higher NL1 expression also displayed severe growth retardation (Figure 4B, 4C, 4F), generally producing only a few leaves with two or three elongated internodes and abnormally developed panicles (Figure 4D and 4E). The effect of ectopic NL1 expression was not limited to the leaves, shoots and panicles, as transgenic plants also displayed abnormal root elongation zones during development. Cell and tissue organization in the root elongation zone of transgenic plants was disorganized compared with that of wild-type plants (Figure $4 \mathrm{G}$ and $4 \mathrm{H}$ ). Therefore, constitutive ectopic $N L 1$ expression inhibits development of various organs and tissues without altering meristem and primordia identities. This result is consistent with the notion that $N L 1$ functions as a monitor and coordinator of organogenesis during panicle development, suggesting that a delicate spatial-temporal $N L 1$ expression pattern is essential for the normal development of rice plants.

Expression of PLA1 and other floral developmental regulators in the nll mutant

In the $n l 1$ mutants, an extra vegetative phytomer and abnormal bracts in the panicle develop, suggesting that vegetative growth is prolonged and floral developmental pathways are affected. We examined whether the expression of several key regulators in floral developmental pathways were affected. RT-PCR analysis of several genes that regulate flowering time, including $O s G I, H d 1$, Hd3a, OsMADS1, OsMADS18 and OsMADS50 [15, 16,
31], showed that only $H d 3 a$ and OsMADS1 expression levels were considerably reduced in the $n l l$ mutant; no detectable difference in expression was observed in the other genes (Figure 6H and 6I). Therefore, we conclude that NL1 regulates the expression of $\mathrm{Hd} 3 \mathrm{a}$ and $\mathrm{Os}$ MADS1.

Since the $n l 1$ mutant shares a similar, but weaker, phenotype with that of pla1, we crossed $n l 1-1$ with trel to investigate any genetic interactions. In the $\mathrm{F}_{2}$ population, a segregation ratio of 189 wild-type plants : $51 \mathrm{nll}$ plants : 73 plal plants was observed, which fits the 9:3:4 ratio $\left(\chi^{2}=2.31, P>0.05\right)$. Double mutants identified by PCR phenocopied single pla1 mutant, suggesting that pla1 is epistatic to $n l 1$. When PLA1 and PLA2 expression levels were examined in the $n l l-3$ mutant by semiquantitative RT-PCR, PLA1 transcription levels were consistently reduced, whereas $P L A 2$ levels were unaffected. In contrast, no detectable difference in $N L 1$ expression was observed between the wild type and the tre 1 mutant (Figure 6G).

The PLAl expression pattern in the $n l 1-3$ mutant was further investigated through RNA in situ hybridization. In the wild-type plant, PLAl was strongly expressed in the bract primordia and incipient bract primordia, with transcripts also detected at the base of vegetative leaves and rachis of the panicle (Figure 6A and 6D). In the $n l 1-3$ mutant, PLA1 was preferentially expressed in the abaxial side of the ectopic bract leaf (Figure 6B). In the developed panicle of the nl1-3 mutant, PLA1 expression was detected only at the base of the small bract primordia and was absent from mature bracts. At the same stage in the wild-type plant, PLA1 transcripts were found within the entire degenerate bract primordium (Figure 6E). Interestingly, NL1 expression was detected on the adaxial side of each bract primordia in the trel mutant, similar to its wild-type expression in the first and second bracts (Figure 6C and 6F). We therefore conclude that the PLA1 expression pattern during panicle development is regulated by NL1.

\section{Discussion}

Here, we explored the model monocot rice plant to investigate the control of organogenesis during reproductive development, unique for monocot architecture and linked to important agronomic traits. One of the most conspicuous morphological processes in rice is sequential UPI elongation concomitant with panicle development. UPIs and other internodes in vegetative phytomers contain the specialized intercalary meristem (IM) found at the base of grass species internodes [4]. UPI elongation stems from both IM cell division and enhanced cell elongation in the zone above the IM [4]. While the 

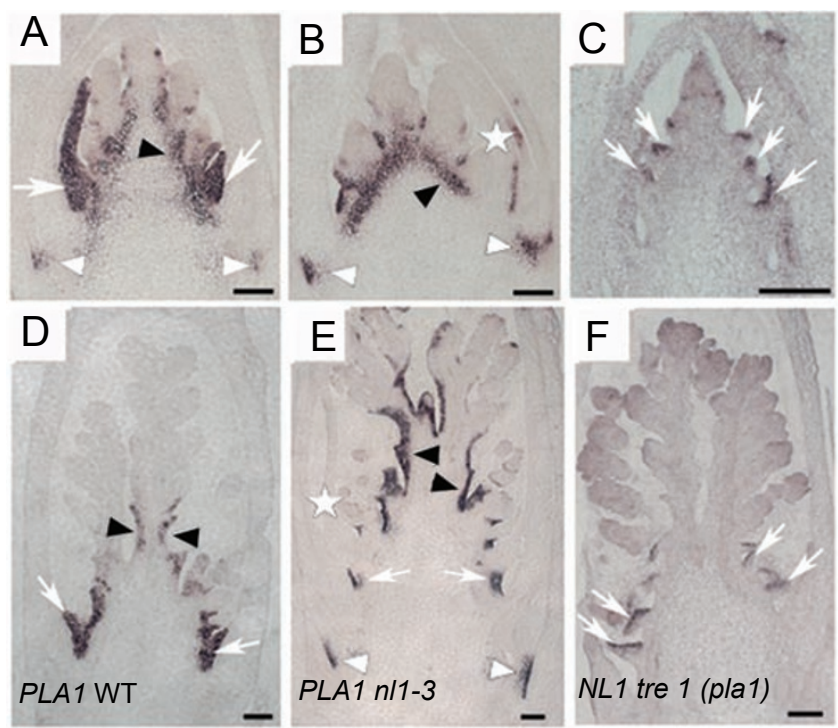

G
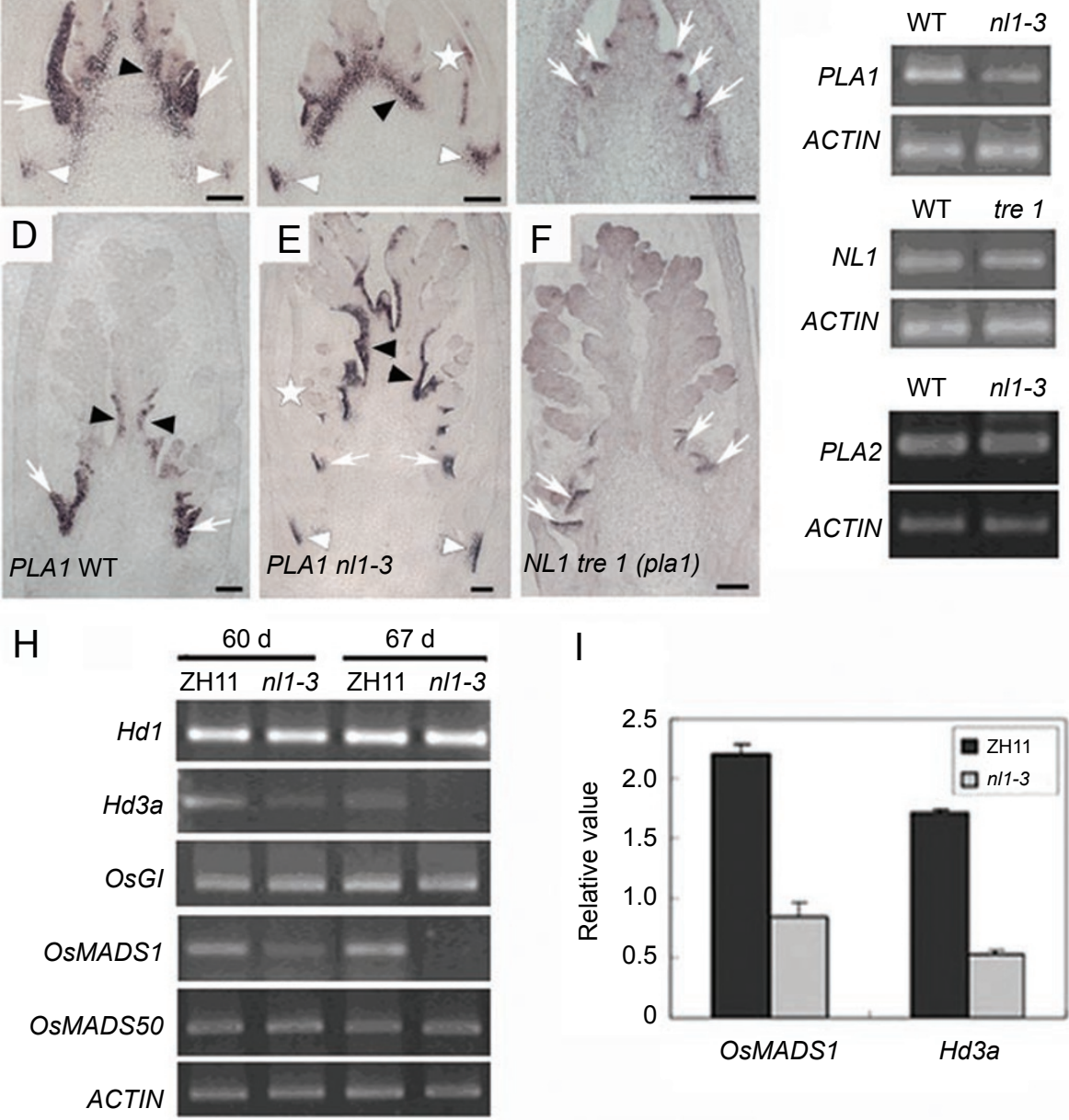

I

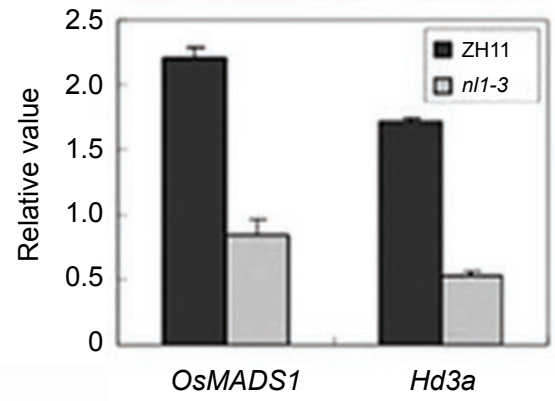

Figure 6 Expression analysis of NL1 and other developmental regulators in different genetic backgrounds. (A-F) Expression pattern of NL1 and PLA1 in wild-type and reciprocal mutant backgrounds. RNA in situ hybridization was conducted in the panicles at the early stage when first branch primordia differentiate (A-C) and at the later stage when stamen and carpel primordia initiate (D-F). Bars $=100 \mu \mathrm{m}$. (A, D) In wild type, PLA1 is expressed throughout the bract primordia at the early stage ( $A$, arrows) and then restrictedly at the bases of bract primordia (D, arrows). PLA1 is also expressed at the base of vegetative leaves (A, white arrowheads) and rachis of panicle (A, D; black arrowheads). (B, E) Altered expression pattern of PLA1 in n/1-3 mutant. PLA1 is expressed abnormally at the abaxial side of the first bract primordia (asterisk) at the early stage (B). At the later stage $(E)$, its expression is confined to the abaxial side on the base of overgrown bract primordia (arrows), and no expression was detected in the more developed bract (asterisk). On the other hand, its expression in the bases of bract primordia (arrows), rachis of panicle (B, E; black arrowheads) and vegetative leaves (B, E; white arrowheads) is not altered. (C, $F$ ) Expression pattern of $N L 1$ is not altered in tre1. NL1 transcripts are detected at the adaxial side of overgrown bract and bract primordia. (G) Semi-quantitative RT-PCR analyses of the PLA1, PLA2 and NL1 expression. cDNA templates were prepared from the inflorescence of wild-type and mutant plants. PCR cycles for amplifying cDNA of PLA1, NL1, PLA2 and ACTIN were 24, 30, 28 and 20, respectively. (H) Semi-quantitative RT-PCR analyses for expression of flowering regulators at 60 and 67 days after germination. Leaf blades of four individual plants (exposed to light after $6 \mathrm{~h}$ ) were collected together as a sample to extract total RNA. Expression of Hd1, Hd3a, OsGI, OsMADS1, OsMADS18 and OsMADS50 (SOC1) were examined. PCR amplification on each sample was performed three times independently. (I) Real-time RT-PCR analysis to quantify the expression level of Hd3a and OsMADS1 in wild type and $n / 1$. Leaf tissues were collected at 60 days after germination.

molecular mechanism controlling IM differentiation and activity merits further investigation, various components of different phytohormone signaling pathways, such as the GA and BR pathways, have been found to play cru- cial roles in the control of UPI elongation [17, 20-23, 25], indicating the involvement of a sophisticated genetic network.

To identify novel components in the control of con- 
comitant growth of both vegetative phytomers and panicles during rice's reproductive development, we analyzed two complementary groups of mutants. Mutant phenotypes included altered UPI elongation patterns, overgrown panicle bracts and asynchrony between panicle development and UPI elongation, as well as prolonged vegetative growth. One locus was found to be allelic to the classical $n l l$ locus and the other to plal. Both NL1 and PLA1 play important roles in the control of organogenesis during reproductive development in rice.

We cloned the NL1 gene, which encoded a GATAlike transcription factor. All GATA factors contain one or more DNA-binding motifs $\left(\mathrm{CX}_{2} \mathrm{CX}_{17} \mathrm{CX}_{2} \mathrm{C}\right.$ in animals and $\mathrm{CX}_{2} \mathrm{CX}_{18} \mathrm{CX}_{2} \mathrm{C}$ in plants) [32], and four classes of GATA factors have thus far been identified in the Arabidopsis and rice genomes. NL1 belongs to class B GATA factors, containing only one conserved DNA-binding motif. In the class B group, four GATA factors in Arabidopsis and three in rice including NL1 (previously identified as OsGATA15) constitute one gene cluster [30]. Four Arabidopsis class A GATA factors were identified as DNA-binding proteins that interact with light-responsive promoters [29]. However, GATA-20, an Arabidopsis class B GATA factor, did not display any specific binding to a light-responsive regulatory element in vitro, suggesting that class B factors may not be directly involved in light signal transduction [33]. The binding motif recognized by class B GATA factors remains to be identified.

NL1 (OsGATA15) is the first GATA factor investigated in rice. In Arabidopsis, the function of one class B GATA factor, HANABA TARANU (HAN) (or At3g50870), has been investigated and found to play an important role in the development of both floral organs and SAMs [34]. han mutants display a reduction of floral organ number and SAM malfunction. In contrast, $n l l$ loss of function gives rise to overgrown bracts and alteration of internode UPI elongation patterns. Therefore, there should be functional divergence between $H A N$ and $N L 1$, which were recruited to different developmental processes in dicots and monocots, respectively, although they have conserved protein domains.

In transgenic rice plants with constitutive $N L 1$ expression, growth retardation, as well as smaller leaves and abnormal root patterns were observed (Figure 4B, 4C, 4G, 4H). Consistently, overexpression of HAN in Arabidopsis also caused growth retardation and SAM malfunction [34], suggesting that a delicate spatial-temporal expression pattern of $N L 1$ and $H A N$ is essential for normal development in both species. NL1 was found to be preferentially expressed in the bract primordia, which degenerate soon after initiation. NL1 may thus indirectly suppress organogenesis when strongly expressed, ac- counting for overgrown bracts in $n l 1$ mutants. In the pla 1 mutant, the predominant accumulation of $N L 1$ transcripts in the bract primordia is unaltered, but overgrown bracts are formed, suggesting that NL1 acts to indirectly suppress bract development and is possibly dependent on PLA1.

NL1 is a GATA-like transcription factor that potentially regulates the expression of other developmental genes. The regulation of PLA1 and PLA2 is independent of each other, and both genes monitor leaf initiation and reproductive development in rice $[10,11] . N L 1$ is preferentially expressed in the bract primordia, where both PLA1 and PLA2 display strong expression. Overall PLA1 expression is reduced and its spatial pattern is also altered in the $n l 1$ mutants (Figure 6A, 6B, 6D, 6E); PLA2 levels remain unaltered (Figure 6G). Additionally, a genetic interaction between $n l l$ and plal was demonstrated with $N L 1$ acting upstream of $P L A 1$. Therefore, it is likely that $P L A 1$ is a downstream target of $N L 1$. However, given that $P L A 1$ mutants display much stronger phenotypes than those of NL1, and that PLA1 expression is reduced, but not abolished, in various $n l 1$ mutants, $N L 1$ may act to modulate or fine-tune PLA1 expression.

As both $H d 3 a$ and OSMADS1 expression is also reduced in the $n l 1$ mutants (Figure $6 \mathrm{H}$ and 6I), PLA1 may not be the only $N L 1$ target. $H d 3 a$ is a key regulator in the control of flowering time, and OsMADS1 is important for floral meristem development [13, 16, 32], which may explain the prolonged vegetative growth observed in the nll mutant with one or two extra vegetative phytomers and delayed heading time. However, NL1 is not preferentially expressed in vegetative leaves and floral organs, where the mRNAs of $H d 3 a$ and OsMADS1, respectively, are most abundantly found. This suggests either that basal $N L 1$ expression is important for the maintenance of $H d 3 a$ and OSMADS1 expression levels or that other regulatory mechanisms exist. In $n l 1$ mutants, overgrown bracts form and ectopic signaling likely occurs, which could in turn indirectly affect $H d 3 a$ and OsMADS1 expression levels. NL1 may thus directly or indirectly regulate vegetative UPI elongation patterns. Therefore, we propose that NL1 acts as both a modulator and a coordinator in the control of vegetative phytomers and panicles during reproductive development in rice.

\section{Materials and Methods}

\section{Plant materials}

Germplasm from rice mutants were screened using our rice mutant screening method, which highlights defects in UPI elongation and panicle patterning. Two complementary groups were identified, one allelic to $n l l$ and the other (tree rice or tre) allelic to plal (Figure 1A). nll-1 was a naturally occurring mutant originally 
identified in the $\mathrm{F}_{2}$ population from a cross between Jiahe 99 and Ningjing 7. nl1-2 was a near-isogenic line carrying a classic mutant locus ( $n l-1)$, generated from more than ten backcrosses of the nl-1 mutant with an indica cultivar Zhefu 802 (ZF802) [26, 35]. nl1-3, tre 1 and tre2, tre3, tre 4 were identified from two M2 populations of japonica cultivar Zhonghua 11 (ZH11) treated with ethyl methanesulfonate and $\gamma$-rays, respectively. Allelic tests confirmed that the $n l 1-1, n l 1-2$ and $n l 1-3$ mutants were allelic to one another. Genetic analyses were conducted to analyze and map the tre locus, which was previously found to be allelic to plastochron1 (pla1) [8].

\section{Molecular cloning of NL1}

The $n l l$ locus was mapped from two $\mathrm{F}_{2}$ populations, crossed between $n l 1-1$ with the two indica varieties Minghui 63 and 9311 , respectively. Of the two $\mathrm{F}_{2}$ populations, 1253 mutants with typically overgrown bracts were used for mapping. All simple sequence repeat (SSR) markers were designed according to the public rice genome sequence in IRGSP (http://rgp.dna.affrc.go.jp/cgibin/statusdb/irgsp-status.cgi) using the SSRIT tool (http://www. gramene.org/db/searches/ssrtool) (Supplementary information, Table S1). The locus was defined as a 7-kb region, and a candidate gene was predicted with the GENESCAN program. This region was directly amplified and sequenced to determine mutations within the candidate gene from three allelic mutants. A genomic DNA fragment containing the $N L 1$ candidate was isolated from the OsJNBa0017N18 BAC clone (accession no. AC120988), and exon and intron regions were determined. For complementation tests, a 6.2-kb fragment (including $3.9 \mathrm{~kb}$ upstream of the start codon and $1.3 \mathrm{~kb}$ of the $3^{\prime}$ end) was ligated into SmaI and HindIII sites within the binary vector pCAMBIA1301 and transformed into Agrobacterium tumefaciens EHA105.

\section{Linkage analysis of tre mutants and double mutant geno- typing}

The tre locus was mapped using four small F2 populations (about 50) generated by crossing tre 1, tre 2, tre 3 , tre 4 with indica cultiva Kasalath, respectively. The polymorphism marker RSL0056 (F: 5'-TCT ATA ATG TAG CCC CCC CC-3', R: 5'TTT CAG GGG CTT CTA CCA AC-3') in the BAC clone OsJNBa0044A10 (AC083943) of chromosome 10 was used to do the linkage analysis with mutants. The results showed that all of the four tre alleles were closely linked with the RSL0056. For all genotyping, nll-1 trel double mutant plants were identified from those that had trel phenotype, by using SL1303 (5'-TCT CCT TCC TCG CCT TCG T-3') and SL1304 (5'-GGC CAC AAT AGT ACG ACTCGG-3') primers.

\section{Scanning Electron Microscopy}

Vegetative SAM and inflorescence meristems were collected and leaves were removed as necessary. Plastic replicas were made and coated with gold-palladium in an E-1010 ion sputter. SEM was performed with a Hitachi S-2460 scanning electron microscope at $15 \mathrm{KV}$. SEM photographs were captured electronically and processed with the Adobe Photoshop 6.0 software.

\section{Histological analysis}

Mature internodes and roots of seedlings were fixed in FAA (formalin : glacial acetic acid : 70\% ethanol $=1: 1: 18, \mathrm{v} / \mathrm{v} / \mathrm{v}$ ) overnight. Internodes were immersed into hydrofluoric acid for 7 days to make them soften. Afterwards, they were dehydrated in a graded ethanol series. After substitution with xylene, the samples were embedded in Paraplast Plus (Sigma) and sectioned at $8 \mu \mathrm{m}$ by using a rotary microtome. Stem sections were stained with $0.05 \%$ $(\mathrm{w} / \mathrm{v})$ toluidine blue $\mathrm{O}$ in sodium phosphate buffer $(100 \mathrm{mM}, \mathrm{pH}$ 7). Root sections were stained with $1 \%(w / v)$ Safranin (water solution) and $0.1 \%(\mathrm{w} / \mathrm{v})$ Fast Green in 95\% ethanol solution and observed with a light microscope (HBO50, ZEISS).

\section{RNA in situ hybridization analysis}

Plant materials were fixed in $4 \%(\mathrm{w} / \mathrm{v})$ paraformaldehyde and $0.25 \%$ glutaraldehyde in $0.1 \mathrm{M}$ sodium phosphate buffer, $\mathrm{pH} 7.4$, overnight at $4{ }^{\circ} \mathrm{C}$, dehydrated through graded ethanol and xylene series, and then embedded in Paraplast Plus (Sigma). Microtome sections $(8 \mu \mathrm{m}$ thick) were applied to glass slides treated with polylysine. To identify the $N L 1$ gene expression, a region consisting of 303-bp coding region and 170-bp 3' UTR region was amplified with the primers $5^{\prime}$-cgg gat cag gta caa gaa gg-3' and 5'-GGT TCG TTT CAA GAT CAG AGT-3' and cloned into pBluescript II KS vector for RNA synthesis and labeling. A 673-bp region amplified with primers 5'-GGC GTT GGT GAC GGA GTG GT-3' and 5'-GCA AGC TCA AGG AGG AAG ACG A-3' was used for PLA1 probe. In situ hybridization with digoxigenin-labeled sense or antisense RNA was conducted according to the method of Coen et al [36].

\section{GUS staining}

The NL1 promoter sequence containing about 2.2-kb fragment just before the start codon was amplified with primers $5^{\prime}$-GAA TTC TCG CTC ATC ACA AGT GGA GG-3' and 5'-CCA TGG CTC CTA TCA ACT GAT GAA C-3', and inserted into the site of EcoRI and NcoI of pSLJ4D4, resulting in $p N L 1-G U S$ protein. The whole fragment was subcloned into the site of EcoRI and HindIII of pCAMBIA1300, resulting in the plasmid pCSL0340. Various tissues of the transgenic plants were submerged in GUS buffer (0.1 M sodium phosphate, $\mathrm{pH} 7.0,0.5 \mathrm{mM}$ potassium ferricyanide, $0.5 \mathrm{mM}$ potassium ferrocyanide, $20 \mathrm{mM}$ EDTA and $100 \mathrm{mg}$ $\mathrm{X}$-Gluc dissolved in $1 \mathrm{ml}$ dimethyl sulfoxide). Then the stained organs were washed by $70 \%$ ethanol to remove the chlorophyll. After they were cleared in the benzyl-benzoatefour-and-a-half fluid devised by Herr [37], the stained organs were observed under a microscope equipped with Nomarski differential interference contrast optics (BX51, DP70; Olympus).

\section{RT-PCR analysis}

Total RNA was extracted from various rice tissues and organs with RNA isolation Reagent Trizol (Bio Basic Inc., Canada). For RT-PCR, first-strand cDNA was synthesized with M-MuLV Reverse Transcriptase (MBI Fermentas). The first-strand cDNA was used as a template, and amplifications were performed for different PCR cycles. To detect different genes' cDNA: primers 5'-CTC TTC TTC CCG ATG TCC A-3' and 5'-CCT TCT CCC CAA CGC TAA T-3' for $N L 1$, 5'-GCC GCC GAC AAT GCC GAC TTC-3' and $5^{\prime}$-TTA GCG TAG GTA AGA CGT GG-3' for PLA1, 5'-ACA AGG CGT TCC ACA AGC AAC C-3' and 5'-CCC AAC ATA ATA TGA TGT GAT AA-3' for PLA2 and 5'-TCC ATC TTG GCA TCT CTC AG-3' and 5'-GTA CCC GCA TCA GGC ATC TG-3' for $A C T I N$ were used. $A C T I N$ was tested as endogenous reference gene. To examine the expressions of flowering-time regulators, 
leaf blades were collected from WT plants and nll-3 mutants grown under $12 \mathrm{~h}$ light/ $12 \mathrm{~h}$ dark conditions. PCR was performed using primers 5'-CCT CAA GGT CAC CTA TGG CTC CAA G-3' and 5'-GAT GAT AGT GAG CAT GCA GCA GAT C-3' for $H d 3 a$ and 5'-TCC ATA TGT CCT GGC AAG AT-3' and 5'-AAG AGA GCA CGC ACG TAC TT-3' for OsMADS1. The primers for Hd1, OSGI, OsMADS50 and OSMADS18 were designed as reported [15]. All PCR experiments were repeated independently two or three times. The real-time RT-PCR reaction was performed using the SYBR ${ }^{\circledR}$ Premix Ex Taq ${ }^{\mathrm{TM}}$ (TaKaRa, Cat. RR041B) on a CORBETT RESEARCH (Australia) Rotor-gene 3000 cycler according to the manufacturer's instructions. ACTIN was used as constitutive control for normalization of candidate gene expression level. A standard curve was drawn using PCR fragment containing the amplified region, and the quantification of each gene was made according to the user manual of CORBETT RESEARCH Rotorgene software. For the real-time PCR, the primers for $H d 3 a$ and OsMADS1 were 5'-GGT CTA CCC CTA GCT AAC GAT GAT C-3', 5'-TGA ACC TGC AAT GTA TAG CAT GCT-3 and 5'-CCA GGC CTA CAT GGA CCA TCT-3', 5'-CGG ATG GGA TGT GTT CAT TG-3', respectively. The primers of $A C T I N$ for real-time RTPCR were 5'-GAA GGA TCT ATA TGG CAA CAT CG-3' and 5'ATC CAC ATC TGC TGG AAT GTG-3'.

\section{$35 S \Sigma N L 1$ overexpression in rice}

A NL1 cDNA was synthesized and amplified from poly $(\mathrm{A})^{+}$ RNA isolated from young inflorescences. A full-length cDNA fragment amplified with the primers 5'-GGG GTA CCA TGC TTC ACC ATT ACT ACA G-3' and 5'-CGG GAT CCG CTA ATG CTA CAC TAG TTA AT-3' was ligated into the KpnI and BamHI sites of a modified pCAMBIA1300 with CaMV 35S promoter as a result of plasmid pCSL0366. For rice transformation, wild-type strain ZH11 was transformed with Agrobacterium tumefaciens strain EHA105 harboring pCSL0366 plasmids.

\section{$G A_{3}$ treatment}

For external $\mathrm{GA}_{3}$ application, $\mathrm{ZH} 11$ and $n l 1-3$ plants grown on soil were sprayed once every 4 days with $\mathrm{GA}_{3}$ when the internode elongation process commenced. $\mathrm{GA}_{3}$ (Sigma) was dissolved in ethanol and used in a final concentration of $10^{-4} \mathrm{M}$.

\section{Acknowledgments}

We thank Dr Hongxuan Lin at the Institute of Plant Physiology and Ecology for the kind support on NL1 mapping. We are grateful to Shuping Xu (Institute of Plant Physiology and Ecology, Chinese Academy of Sciences) and Meixian Yan (China National Rice Research Institute) for the help of transgenic work, and we acknowledge financial support of National High-Tech Projects from the Ministry of Science and Technology of China (grant number: 2006AA10A102).

\section{References}

1 Poethig RS. Phase change and the regulation of shoot morphogenesis in plants. Science 1990; 250:923-930.

2 Poethig RS. Phase change and the regulation of developmental timing in plants. Science 2003; 301:334-336.
3 Sussex IM, Kerk NM. The evolution of plant architecture. Curr Opin Plant Biol 2001; 4:33-37.

4 Hoshikawa $\mathrm{K}$. The growing rice plant: an anatomical monograph. Tokyo: Nobunkyo, 1989.

5 Itoh J, Nonomura KI, Ikeda K, et al. Rice plant development: from zygote to spikelet. Plant Cell Physiol 2005; 46:23-47.

6 Wang Y, Li J. The plant architecture of rice (Oryza sativa L.). Plant Mol Biol 2005; 59:75-84.

7 Asai K, Satoh N, Sasaki H, Satoh H, Nagato Y. A rice heterochronic mutant, mori1, is defective in the juvenile-adult phase change. Development 2002; 129:265-273.

8 Itoh JI, Hasegawa A, Kitano H, Nagato Y. A recessive heterochronic mutation plastochron 1 shortens the plastochron and elongates the vegetative phase in rice. Plant Cell 1998; 10:1511-1521.

9 Ahn O, Miyoshi K, Itoh JI, Nagato Y, Kurata N. A genetic and physical map of the region containing PLASTOCHRON1, a heterochronic gene, in rice (Oryza sativa L.). Theor Appl Genet 2002; 105:654-659.

10 Miyoshi K, Ahn B-O, Kawakatsu T, et al. PLASTOCHRON1, a timekeeper of leaf initiation in rice, encodes cytochrome P450. Proc Natl Acad Sci USA 2004; 101:875-880.

11 Kawakatsu T, Itoh JI, Miyoshi K, et al. PLASTOCHRON2 regulates leaf initiation and maturation in rice. Plant Cell 2006; 18:612-625.

12 Yano M, Katayose Y, Ashikari M, et al. Hd1, a major photoperiod sensitivity quantitative trait locus in rice, is closely related to the Arabidopsis flowering time gene CONSTANS. Plant Cell 2000; 12:2473-2484.

13 Hayama R, Yokoi S, Tamaki S, Yano M, Shimamoto K. Adaptation of photoperiodic control pathways produces short-day flowering in rice. Nature 2003; 422:719-722.

14 Doi K, Izawa T, Fuse T, et al. Ehd1, a B-type response regulator in rice, confers short-day promotion of flowering and controls FT-like gene expression independently of Hdl. Genes Dev 2004; 18:926-936.

15 Lee S, Kim J, Han JJ, Han MJ, An G. Functional analyses of the flowering time gene OSMADS50, the putative SUPPRESSOR OF OVEREXPRESSION OF COI/AGAMOUS-LIKE 20 (SOC1/AGL20) ortholog in rice. Plant J 2004; 38:754-764.

16 Chung YY, Kim SR, Finkel D, Yanofsky MF, An G. Early flowering and reduced apical dominance result from ectopic expression of a rice MADS box gene. Plant Mol Biol 1994; 26:657-65.

17 Yamamuro $\mathrm{C}$, Ihara $\mathrm{Y}, \mathrm{Wu} \mathrm{X}$, et al. Loss of function of a rice brassinosteroid insensitivel homolog prevents internode elongation and bending of the lamina joint. Plant Cell 2000; 12:1591-1605.

18 Takeda K. Internode elongation and dwarfism in some gramineous plants. Gamma Field Sym 1977; 16:1-18.

19 Sasaki A, Ashikari M, Ueguchi-Tanaka M, et al. Green revolution: a mutant gibberellin-synthesis gene in rice. Nature 2002; 416:701-712.

20 Hong Z, Ueguchi-Tanaka M, Umemura $\mathrm{K}$, et al. A rice brassinosteroid-deficient mutant, ebisu dwarf (d2), is caused by a loss of function of a new member of cytochrome P450. Plant Cell 2003; 15:2900-2910.

21 Hong Z, Ueguchi-Tanaka M, Fujioka S, et al. The Rice brassinosteroid-deficient dwarf 2 mutant, defective in the rice 
homolog of Arabidopsis DIMINUTO/DWARF1, is rescued by the endogenously accumulated alternative bioactive brassinosteroid, dolichosterone. Plant Cell 2005; 17:2243-2254.

22 Tanabe S, Ashikari M, Fujioka S, et al. A novel cytochrome P450 is implicated in brassinosteroid biosynthesis via the characterization of a rice dwarf mutant, $d$ warf11, with reduced seed length. Plant Cell 2005; 17:776-790.

23 Zhu Y, Nomura T, Xu Y, et al. ELONGATED UPPERMOST INTERNODE encodes a cytochrome P450 monooxygenase that epoxidizes gibberellins in a novel deactivation reaction in rice. Plant Cell 2006; 18:442-456.

24 Sakamoto T, Miura K, Itoh H, et al. An overview of gibberellin metabolism enzyme genes and their related mutants in rice. Plant Physiol 2004; 134:1642-1653.

25 Sato Y, Sentoku N, Miura Y, Hirochika H, Kitano H, Matsuoka M. Loss-of-function mutations in the rice homeobox gene OSH15 affects the architecture of internodes resulting in dwarf plants. EMBO J 1999; 18:992-1002.

26 Nagao S, Takahashi M. Trial construction of twelve linkage groups in Japanese rice. Genetical studies on rice plant, XXVII. J Fac Agric Hokkaido Univ, 1963; 53:72-130.

27 Luo A, Qian Q, Yin H, et al. EUI1, encoding a putative cytochrome P450 monooxygenase, regulates internode elongation by modulating gibberellin responses in rice. Plant Cell Physiol 2006; 47:181-191.

28 Kikuchi K, Terauchi K, Wada M, Hirano H-Y. The plant MITE mPing is mobilized in anther culture. Nature 2003; 421:167-170.

29 Teakle GR, Manfield IW, Graham JF, Gilmartin PM. Arabidopsis thaliana GATA factors: organisation, expression and
DNA-binding characteristics. Plant Mol Biol 2002; 50:43-57.

30 Reyes JC, Muro-Pastor MI, Florencio FJ. The GATA family of transcription factors in Arabidopsis and rice. Plant Physiol 2004; 134:1718-1732.

31 Agrawal GK, Abe K, Yamazaki M, Miyao A, Hirochika H. Conservation of the E-function for floral organ identity in rice revealed by the analysis of tissue culture-induced loss-offunction mutants of the OsMADS1 gene. Plant Mol Biol 2005; 59:125-135.

32 Teakle GR, Gilmartin PM. Two forms of type IV zinc-finger motif and their kingdom-specific distribution between the flora, fauna and fungi. Trends Biochem Sci 1998; 23:100-102.

33 Jeong MJ, Shih MC. Interaction of a GATA factor with cisacting elements involved in light regulation of nuclear genes encoding chloroplast glyceraldehyde-3-phosphate dehydrogenase in Arabidopsis. Biochem Biophys Res Commun 2003; 300:555-562.

34 Zhao Y, Medrano L, Ohashi K, et al. HANABA TARANU is a GATA transcription factor that regulates shoot apical meristem and flower development in Arabidopsis. Plant Cell 2004; 16:2586-2600.

35 Zeng D, Qian Q, Dong G, et al. Development of isogenic lines of morphological markers in Indica rice. Acta Bot Sin 2003; 45:1116-1120.

36 Coen ES, Romero JM, Doyle S, Elliott R, Murphy G, Carpenter R. Floricaula: a homeotic gene required for flower development in antirrhinum majus. Cell 1990; 63:1311-1322.

37 Herr JM Jr. An analysis of methods for permanently mounting ovules cleared in four-and-a-half type clearing fluids. Stain Technol 1982; 57:161-169.

(Supplementary information is linked to the online version of the paper on the Cell Research website.) 